\title{
tic\&société
}

Vol. 10, N 2-3 | 2ème semestre 2016 - 1er semestre 2017

Spécial varia

\section{Une question de comportement. Recommandation des contenus audiovisuels et transformations numériques}

Joëlle FARCHY, Cécile MÉADEL et Arnaud ANCIAUX

\section{OpenEdition \\ Journals}

Édition électronique

URL : http://journals.openedition.org/ticetsociete/2136

DOI : 10.4000/ticetsociete.2136

Éditeur

Association ARTIC

Édition imprimée

Pagination : 168-198

Référence électronique

Joëlle FARCHY, Cécile MÉADEL et Arnaud ANCIAUX, « Une question de comportement.

Recommandation des contenus audiovisuels et transformations numériques », tic\&société [En ligne],

Vol. 10, № 2-3 | 2ème semestre 2016 - 1er semestre 2017, mis en ligne le 30 avril 2017, consulté le 30 avril 2019. URL : http://journals.openedition.org/ticetsociete/2136; DOI : 10.4000/ticetsociete.2136 
tic\&société - 10(2-3), 2016-2017

\title{
Une question de comportement Recommandation des contenus audiovisuels et transformations numériques
}

\author{
Joëlle FARCHY, Cécile MÉADEL, Arnaud ANCIAUX
}

Joëlle Farchy est économiste, professeure à l'université Paris 1, Panthéon-Sorbonne où elle dirige l'École des médias et du numérique de la Sorbonne. Elle est en outre personnalité qualifiée au Conseil supérieur de la propriété littéraire et artistique (CSPLA) et commissaire à la Commission nationale de l'informatique et des libertés (CNIL). Ses travaux portent sur l'économie numérique, les industries culturelles, la propriété intellectuelle et la régulation de I'Internet. Joelle.Farchy@univ-paris1.fr

Cécile Méadel est sociologue, professeure à l'université PanthéonAssas, membre du CARISM, chercheuse associée au Centre de sociologie de l'innovation (Mines ParisTech - CNRS). Ses travaux portent sur les technologies de l'information, du point de vue de la construction des usages, de la genèse des dispositifs et de la mise en forme des usagers, des clients, des amateurs. Ces dernières années, elle a travaillé sur la régulation de l'Internet (du Bitcoin aux e.communautés) et sur la configuration industrielle des publics (à travers des outils de quantification ou des dispositifs de recommandation). cecile.meadel@u-paris2.fr

Arnaud Anciaux est professeur au Département d'information et de communication de l'Université Laval et membre du Centre de recherche interuniversitaire sur la communication, l'information et la société $(\mathrm{CRICIS})$. Ses travaux actuels s'attachent à croiser 


\section{Joëlle FARCHY, Cécile MÉADEL, Arnaud ANCIAUX}

approche économique, étude sociologique et analyse de discours pour comprendre les transformations contemporaines des industries et des marchés de la culture et de la communication. arnaud.anciaux@com.ulaval.ca 
Une question de comportement. Recommandation des contenus audiovisuels et transformations numériques

\section{Une question de comportement Recommandation des contenus audiovisuels et transformations numériques}

Résumé : Parmi l'ensemble des dispositifs visant à orienter l'internaute au sein d'une offre audiovisuelle pléthorique, nous distinguons ceux qui s'appuient sur les jugements et ceux fondés sur l'analyse des comportements. L'étude de certains dispositifs proposés en France montre que les recommandations personnalisées, fondées sur l'analyse détaillée des comportements des internautes, parce qu'elles se révèlent d'une efficacité redoutable, occupent une place de plus en plus centrale. Chacune à leur manière, les diverses formes de recommandation posent aux opérateurs de mise à disposition de contenus audiovisuels en ligne de nouveaux défis qui nécessiteront à l'avenir de tester des formes de différenciation concurrentielle. La recommandation personnalisée algorithmique, en particulier, entraîne d'importantes transformations des modèles économiques, des restructurations des marchés et des renouvellements des métiers.

Mots-clés: marché audiovisuel, recommandation, algorithme, contribution des usages, prescription.

Abstract: This article focuses on the various apparatus that guide the Internet user confronted with an ample broadcasting supply; it distinguishes between apparatus based on judgments and apparatus based on behaviour. It highlights the fact that the second type are becoming more and more important given their amazing efficiency due to precise analysis of the users' behaviour. Each in its own manner, various forms of recommendation pose challenges to audio-visual online operators who will need to respond with new ideas for competitive differentiation. The personalised algorithmic recommendation, in particular, is transforming economic models, market structures and the organisation of professions.

Keywords: audiovisual market, recommendation, prescription, algorithm, user's contribution. 
Resumen: Entre todos los dispositivos cuyo objetivo es orientar a los internautas dentro de una inmensa oferta audiovisual, cabe distinguir los dispositivos que dependen de los juicios y los que están basados en el análisis del comportamiento. El estudio de ciertos dispositivos que se ofrecen en Francia, muestra que las recomendaciones personalizadas, basadas en un análisis detallado del comportamiento de los usuarios de Internet, que han resultado ser extremadamente eficaces, adquieren cada vez más importancia. Cada una a su manera, las diferentes formas de recomendación plantean, a los operadores de contenidos audiovisuales en línea, nuevos retos que requerirán poner a prueba nuevas formas de diferenciación competitivas. La recomendación personalizada algorítmica, en particular, provoca cambios profundos en los modelos de negocio, en la reestructuración de los mercados y en la renovación de los oficios.

Palabras clave : mercado audiovisual, recomendación, algoritmo, usos, prescripción. 


\section{Une question de comportement. Recommandation des contenus audiovisuels et transformations numériques}

\section{Introduction}

Comment sont choisis les biens ou les services dans un univers d'abondance informationnelle où l'intérêt du consommateur est la ressource rare face à une offre pléthorique ? Cette question, formulée bien avant l'essor d'Internet en termes "d'économie de l'attention " (Simon, 1971), est devenue un enjeu central sur les marchés numériques. Suivant l'économie des qualités (Callon, Méadel et Rabeharisoa, 2000), la question peut aussi être abordée en fonction du travail de qualification conduit par les acteurs pour permettre le choix des consommateurs : tout marché nécessite en effet que soient précisées les caractéristiques des produits, retravaillées en permanence en fonction des clients visés.

Sur le marché de l'audiovisuel, depuis plus de trente ans, avec les premières générations de réseaux de vidéocommunication des années 1980 (Akrich et Méadel, 2006), différentes applications s'emploient à diversifier les voies d'accès aux programmes audiovisuels, diversification très largement amplifiée par la diffusion de l'ADSL ${ }^{1}$. Toute une galaxie d'acteurs numériques propose désormais des contenus, à l'unité ou par abonnement, suivant des modalités légales ou pas ${ }^{2}$, provenant de professionnels (notamment VOD ${ }^{3}$ ) ou d'amateurs $\left(\mathrm{UGC}^{4}\right)$, avec un statut d'hébergeur (YouTube) ou d'éditeurs (Canal+, TF1, Orange, Netflix, etc.). Le taux de pénétration en France de la télévision de rattrapage ${ }^{5}$ est passé de $54,3 \%$ fin 2010 à $80,1 \%$ lors des neuf premiers mois de $2016^{6}$ et celui de la VOD payante (location d'un

1 L'ADSL (pour Asymmetric Digital Subscriber Line) utilise le réseau téléphonique pour transmettre des données numériques par une transmission dite " à haut débit ".

2 Les formes de mise à disposition de contenus audiovisuels sans l'autorisation des ayants droit, qui soulèvent des questions spécifiques, ne seront pas traitées dans le cadre de cet article.

${ }^{3}$ Video On Demand.

${ }^{4}$ User Generated Contents.

${ }^{5}$ Ou Catch-up TV : il s'agit d'un service proposant de voir ou revoir un contenu, diffusé sur la chaîne de manière linéaire, pendant une période déterminée après leur diffusion.

${ }^{6}$ Baromètre de la télévision de rattrapage, septembre 2016, CENTRE NATIONAL DU CINEMA ET DE L'IMAGE ANIMEE (CNC), en ligne. Ce taux de pénétration s'élevait à $72,6 \%$ le trimestre précédant les entretiens réalisés. 
contenu, achat ou abonnement au service), de $18,6 \%$ en 2009 à $34,4 \%$ en $2015^{7}$. Ces consommations peuvent se faire par l'entremise du support offert par le téléviseur (et des offres dites triple-play), tout particulièrement pour la VOD payante ${ }^{8}$.

La démultiplication des contenus disponibles n'entraîne pas nécessairement une diversification des consommations. Comme le montrent Beuscart, Beauvisage et Maillard (2012) à propos de la télévision de rattrapage, "les formats de consommation numérique ont également des propriétés susceptibles de générer non pas de la dispersion, mais des fortes concentrations de l'attention collective " (p. 46), avec des audiences "globalement synchronisées ". C'est donc bien que des mécanismes sont en œuvre, qui orientent la consommation vers des contenus particuliers. Le "buzz » joue sans doute un rôle central, mais il s'accompagne d'un ensemble d'autres dispositifs de recommandation que nous nous proposons d'étudier.

L'offre de programmes se présente dans un catalogue de contenus que doit construire l'opérateur, et que celui-ci accompagne de moyens d'orientation et de repérage. Ces dispositifs qui visent à promouvoir certains contenus ne sont pas uniquement produits par les opérateurs; d'autres acteurs, et au premier chef les consommateurs, s'emploient à orienter, décrire, apprécier, valoriser, critiquer, noter, etc., les contenus et à le faire savoir. Nous utilisons le terme de recommandation pour désigner l'ensemble de ces dispositifs visant à orienter l'internaute vers un contenu ou un ensemble de contenus particuliers. D'un point de vue économique, la recommandation facilite l'appariement de l'offre et de la demande sur des marchés de prototypes où existent de fortes asymétries d'information. Son objectif principal est de réaliser des propositions, pouvant être considérées comme étant

\footnotetext{
${ }^{7}$ Public de la vidéo à la demande, Ressources statistiques, CNC, mai 2016.

${ }^{8}$ Pour la télévision de rattrapage, le CNC estime pour l'année 2015 qu'il y a eu 1946,75 millions de vidéos de télévision en ligne vues sur ordinateur, 2046,13 vues sur téléviseur, et 1853,21 vues sur un appareil mobile. En revanche, pour la VOD payante en $2015,84,1 \%$ des consommateurs l'ont fait par téléviseur, mais seulement $35,2 \%$ par leur ordinateur et $23,7 \%$ sur un appareil mobile.
} 
Une question de comportement. Recommandation des contenus audiovisuels et transformations numériques

cohérentes par le consommateur, afin de l'inciter à réaliser un acte d'achat ou de location pour les plateformes payantes à l'acte, à augmenter sa satisfaction et l'encourager à demeurer auprès du service pour les plateformes demandant un abonnement ou, pour celles qui sont gratuites, à accroître l'audience du site, source de revenus publicitaires. La recommandation permet d'améliorer et de maximiser l' $A R P U^{9}$ dans les cas où le consommateur ne recherche pas un contenu précis et déterminé.

La transformation des formats de consommation de contenus accompagne et entraîne des restructurations du marché (Anciaux, 2014 ; Farchy et Moreau, 2016). Les différents médias, tels que la presse imprimée et l'audiovisuel, sont marqués par des difficultés grandissantes à entretenir un modèle d'affaires reposant sur des marchés à plusieurs versants (Rochet et Tirole, 2003). De nouveaux acteurs, dont l'emblématique plateforme transnationale Netflix, ont créé des offres, contournant les éventuelles barrières à l'entrée dans l'édition et la distribution de chaînes de télévision. Pour les acteurs de la télévision linéaire, la concurrence sur le marché de la distribution de contenus auprès des consommateurs finaux en est accrue. Cette compétition se construit dans deux directions, concourant à diminuer la rentabilité des modèles d'affaires. En premier lieu, le temps disponible des consommateurs est potentiellement fragmenté entre les propositions d'un très grand nombre d'acteurs (Cha et ChanOlmsted, 2012 ; Lee, Hong et Lee, 2010). En second lieu, et tout particulièrement pour la télévision payante, c'est également la disponibilité à payer du consommateur qui peut être déplacée au profit de nouvelles offres, qu'il s'agisse de paiements à l'acte ou d'abonnements. Face à cela, les acteurs traditionnels de l'audiovisuel sont incités à adapter leurs offres, si ce n'est enjoints à se transformer radicalement dans leur manière d'orienter l'usager.

9 Average revenue per user: mesure utilisée pour déterminer le revenu par consommateur. 
Pour analyser ces bouleversements, nous commençons par proposer une cartographie des différents formats de recommandation qui nous conduit à distinguer quatre grandes formes : d'un côté, les recommandations éditoriale et contributive, qui s'appuient sur les jugements des internautes ou des professionnels; d'un autre côté, les recommandations autoentretenue et personnalisée, fondées toutes deux sur l'analyse des comportements des internautes. Nous examinons ensuite plus spécifiquement certains des dispositifs proposés en France, pour analyser, en conclusion, les principaux enjeux du point de vue des offreurs, en matière de restructurations des marchés et de renouvellement des métiers. Cette recherche s'appuie sur une analyse de la littérature et de la documentation professionnelle, mais aussi, pour la deuxième partie, sur des rencontres avec des professionnels ${ }^{10}$.

\section{CartograpEie des dispositifs de recommandation}

Les professionnels de l'audiovisuel distinguent généralement trois niveaux de recommandation (Patino, 2016 ; Scherer, 2015) :

«Dans le contrôle de l'accès, la programmation éditoriale va se poursuivre, surtout pour trier dans le bruit de la profusion de contenus, pour contextualiser et pour agréger des contenus qui auront du mal à survivre seuls. Le public ne veut plus davantage de choix, mais mieux trouver les contenus et les œuvres pertinentes, et de la manière la plus simple possible. Mais sur le sofa virtuel, face à la tyrannie du choix, les recommandations sociales piloteront aussi cette nouvelle consommation à la demande, avec le search. Tout comme les recommandations des algoritEmes » (Scherer, 2015, p. 25).

Au-delà de ces trois types, de nombreux dispositifs mêlent outils automatiques et traitement humain, où la prégnance respective des différentes modalités de recommandation varie selon les dispositifs envisagés. Nous les avons visualisés dans une cartographie originale (Figure 1) selon deux axes de définition. Le premier axe (DO) décline le dispositif en fonction de sa proximité

\footnotetext{
10 Les auteurs remercient également les deux rapporteurs anonymes de la revue pour leur lecture attentive et leurs critiques bienveillantes et constructives.
}

tic\&société - 10(2-3), 2016-2017 
Une question de comportement. Recommandation des contenus audiovisuels et transformations numériques

avec l'offre ou la demande : à un extrême, des recommandations entièrement "industrielles ", à l'autre, celles émanant de consommateurs ou d'usagers. Les conceptions et les constructions de l'offre et de la demande s'entrelacent cependant bien souvent; ainsi, la recommandation d'un consommateur de série sur le site d'un diffuseur est une production émanant de la demande, mais largement préconstruite, encadrée ou valorisée par le diffuseur. Le second axe (CJ) oppose la matière de la recommandation selon qu'elle exprime un jugement assumé ou qu'elle reflète un comportement qu'il s'agit de capturer. À partir de ces deux axes, quatre modalités de recommandation peuvent schématiquement être distinguées: éditoriale, contributive, autoentretenue et personnalisée.

Figure 1 : Diagramme des recommandations

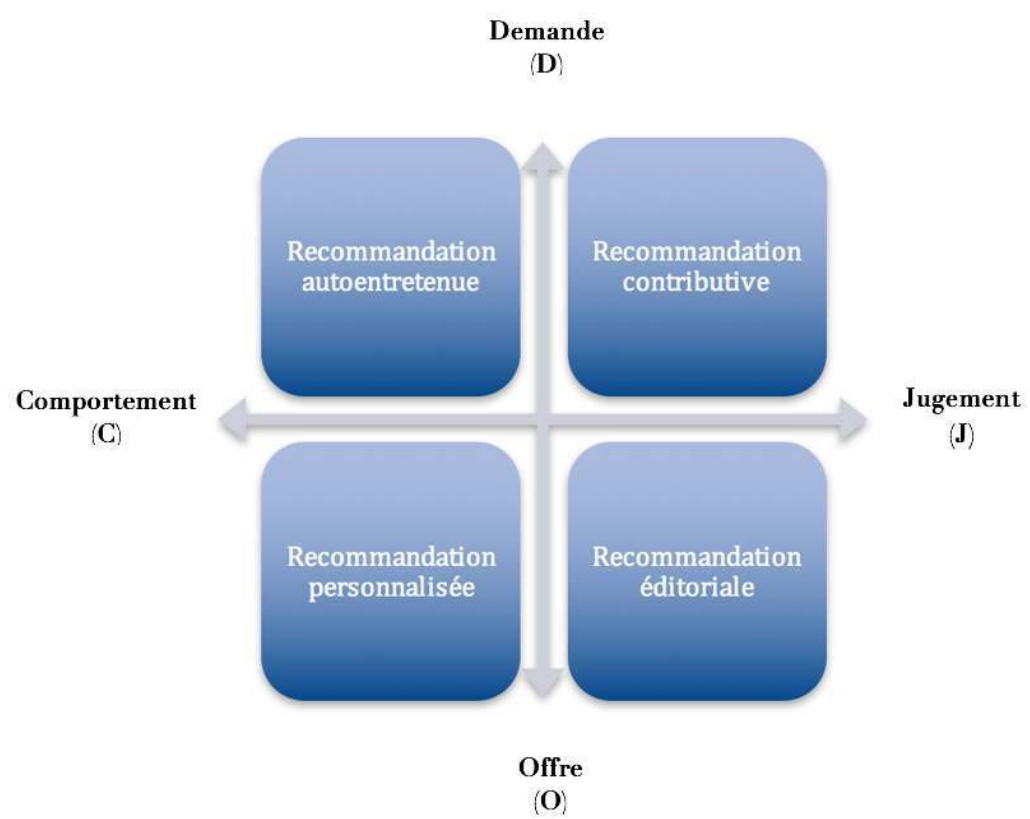


Dans la réalité, la plupart des dispositifs allient jugements et comportements, logiques industrielles et contributions des internautes. Ainsi, dans l'application Zoom pour iPhone lancée par France Télévisions en 2015, l'utilisateur indique d'abord ses centres d'intérêt (culture, sport, jeux) et les émissions qu'il ne veut pas voir. La chaîne publique lui propose ensuite, pour un visionnage sur téléphone mobile, des playlists thématiques de ses émissions dont la sélection s'affine progressivement en fonction du comportement du consommateur.

Nous qualifions de recommandation éditoriale la proposition spécifique de certains contenus fondée sur la mention manifeste ou à tout le moins reconnue et reconnaissable - d'un choix assumé de l'entreprise dans le dispositif (quadrant JO). La fonction éditorialisée (critiques professionnelles, productions marketing, etc.) est souvent distinguée d'une fonction peu ou prou automatisée (suggestions, démarche algorithmique, etc.). Autrement dit, l'entreprise met en avant ou sélectionne explicitement des contenus, au même titre qu'elle pourrait le faire sur une chaîne diffusée de manière linéaire, et elle construit un certain nombre d'énoncés, formulés en direction de l'utilisateur.

Par ailleurs, sur la base de l'activité des internautes, se sont construites les formes d'une recommandation contributive reposant non sur une prise de parole et un jugement explicite de l'entreprise gérant le dispositif, mais sur la mise en valeur des internautes ${ }^{11}$ (quadrant JD). II s'agit là des modalités traditionnelles de la prescription de contenus culturels, comme le bouche-àoreille, qui permettent de renforcer leur visibilité, de contribuer à une meilleure connaissance de biens fortement marqués par leur nature expérientielle et de susciter ainsi une certaine confiance. Dans ces dispositifs, les internautes expriment un véritable jugement. Les systèmes proposent parfois des interventions

\footnotetext{
11 Dans la réalité, la distinction entre les deux formes est parfois délicate, comme en témoigne le nombre de faux avis de consommateurs sur Internet alimentés par des commerçants. L'importance de ce phénomène, que certains évaluent de 10 à $30 \%$ des avis de consommateurs sur Internet (Reagle, 2015), a conduit à un travail de normes et de labellisation dans plusieurs secteurs, comme la norme Afnor du tourisme.
} 
Une question de comportement. Recommandation des contenus audiovisuels et transformations numériques

impliquantes, " une dimension expressive " (la rédaction libre d'un avis), parfois une simple "dimension arithmétique " (l'agrégation de notes) (Beauvisage et al., 2013, p. 13). Partout, l'internaute est à l'œuvre au centre des dispositifs qui encouragent, encadrent et louent sa présence. Depuis le milieu des années 2000, la promotion du Web 2.0 salue les prises de paroles des internautes ainsi que leur capacité à créer, à fabriquer et à reconstruire les offres de biens et services. Et si ces pratiques semblent rares parmi les usagers d'Internet, elles n'en sont pas pour autant mineures (Rebillard, 2011). Cette capacité repose certes sur des racines anciennes, mais elle trouve de nouveaux ressorts dans l'environnement numérique grâce à la multiplication des dispositifs encourageant la contribution et la participation (Dujarier, 2008).

Partout, les internautes sont au travail ; parfois, ce travail est de si faible intensité que l'internaute s'aperçoit à peine qu'il apporte sa contribution à une œuvre collective; parfois, au contraire, l'investissement est massif, intense. L'internaute peut se voir mobilisé bien au-delà de ses contributions et de ses interventions actives et volontaires comme une figure centrale d'une économie numérique en voie de développement (Assogba et al., 2016). Au cœur de l'économie numérique se trouvent en effet la collecte et l'exploitation de données obtenues grâce au "travail » de la "multitude» des internautes (Verdier et Colin, 2012). Les utilisateurs, bénéficiaires de services rendus, deviennent des collaborateurs bénévoles. Ce modèle de la gratuité coopérative, qui a suscité l'enthousiasme de Bekler (2006) et d'autres économistes comme alternative à la coordination par les prix, est en réalité hybride et mélange du marchand, du public et du don (Farchy, Méadel et Sire, 2015). Ce travail gratuit fait écho aux théories du capitalisme cognitif (Moulier-Boutang, 2010) et correspond à une économie de pollinisation et de contribution. II fait également écho au courant critique, sur le Digital Labor, porté par des chercheurs qui analysent la dimension d'exploitation à l'œuvre dans ce travail (Cardon et Casilli, 2015 ; Scholz, 2013), intégré dans de nouvelles formes et déploiements du capitalisme (Terranova, 2000).

Le rapport Colin et Collin (2013) distingue trois catégories de données provenant des internautes : 1) les données soumises par 
l'utilisateur, qui ont fait l'objet d'une saisie explicite par ce dernier, pour différents motifs ; 2 ) les données observées, qui résultent du recueil de traces d'utilisation d'une application; 3) et les données inférées déduites de traitements, en particulier de recoupements, pratiquées par une entreprise à partir de données personnelles de ses utilisateurs et clients. Seule la première catégorie, c'est-à-dire les données qui impliquent une participation délibérée de la part de l'internaute, représente des contributions actives (Méadel, Musiani et Farchy, 2016) et participe de la recommandation que nous avons qualifiée de contributive.

Dans d'autres dispositifs, les internautes laissent quelques traces, plus ou moins consciemment. Nous nommons recommandation autoentretenue les dispositifs qui ne s'appuient pas sur des jugements, élaborés (critique d'une émission) ou simplistes (likes, étoiles) des internautes, mais sur les comportements des uns qui, une fois agrégés, servent de boussole à leurs pairs (quadrant $C D$ ).

Les comportements des internautes ont également conduit à l'émergence d'une nouvelle forme de recommandation souvent qualifiée de moteur de recommandation (Amatriain et Basilico, 2015 ; Ricci, Rokach et Shapira, 2015). La notion, associée à l'automatisme des machines et opposée à l'intervention humaine, repose sur les potentialités techniques d'Internet et sur l'étude des comportements grâce à l'exploitation de données (Gomez-Uribe et Hunt, 2015), permettant de redessiner les interfaces et les dispositifs qui présentent les catalogues de contenus (Elkhatib et al., 2014 ; Machado Vieira et Correia Queiroz, 2015). Cet accompagnement de plus en plus sophistiqué permet de guider l'usager au sein d'une offre abondante, au profit de modèles d'affaires spécifiques (Resnick et Varian, 1997). Pour qualifier ces dispositifs, au terme de recommandation algorithmique souvent utilisé, nous préférons celui de recommandation personnalisée (quadrant $\mathrm{CO}$ ) pour souligner que la nouveauté réside moins dans la prouesse technique que dans les résultats sophistiqués et individualisés qu'elle permet d'obtenir.

Au cœur de nombreux discours, espérances et craintes, ces modalités de recommandation reposent sur les parcours des 
Une question de comportement. Recommandation des contenus audiovisuels et transformations numériques

consommateurs. Elles comportent la promesse de pouvoir leur proposer, par différents moyens, des contenus supposés les intéresser, et ainsi d'améliorer in fine la pertinence réelle ou perçue du service. Nous dirons donc d'une recommandation qu'elle est personnalisée quand elle est construite sur le fondement des informations et des parcours de l'utilisateur. Étape presque finale d'un processus de délinéarisation et d'individualisation, le consommateur devient en quelque sorte à la fois sujet et objet du discours porté par le dispositif. Ce sont ses propres comportements déclarés ou observés et analysés par un opérateur extérieur qui forment le matériau d'une démarche prédictive dans des dispositifs industrialisés.

La recommandation consiste tout d'abord en la proposition de contenus liés à ceux que le consommateur est en train de consulter. Reprenant un principe développé avec succès par la plateforme Amazon sur le modèle du filtrage collaboratif, les recommandations sont effectuées en comparant le profil de l'internaute à ceux d'autres utilisateurs ayant acheté le même produit: " les internautes qui ont acheté le livre que vous achetez ont également acheté ceux-ci ». Le service de recommandation de Netflix, lui aussi présenté comme une référence par les analystes ou les professionnels, est hautement revendiqué comme modèle de recommandation algorithmique par l'entreprise elle-même (Tryon, 2015), qui se targue de ce que $75 \%$ des contenus visionnés par ses clients viendraient d'une recommandation personnalisée de son moteur. Pourtant, le dispositif proposé par Netflix est plus hybride qu'il n'y paraît, puisqu'il mixe une recommandation algorithmique automatisée et une intervention humaine de qualification des contenus et des utilisateurs. La finesse des micro-genres de classement des contenus illustre la mosaïque des cibles marketing visées et la volonté d'améliorer l'expérience de chacun. Pas moins de 77000 « genres " ont ainsi été répertoriés (Madrigal, 2014) comme les «thrillers violents au sujet des chats pour les 8-10 ans ». Cette qualification suppose la visualisation de chaque contenu par un salarié de l'entreprise qui, après avoir tagué ses composantes, proposera une catégorisation 
susceptible d'être croisée avec les comportements des utilisateurs $^{12}$. Dès lors, la puissance du modèle économique de la plateforme n'est pas liée à la seule force technique de son algorithme, mais à l'intensité et à la qualité des données collectées sur les utilisateurs eux-mêmes. La puissance de la recommandation personnalisée repose donc essentiellement sur les comportements de l'ensemble des utilisateurs scrutés en permanence par des opérateurs.

\section{Le réalisme efficace de l'étude des comportements}

Le travail mené dans cette partie s'appuie sur des entretiens réalisés en 2015 avec une dizaine de responsables stratégiques travaillant dans les principaux groupes de l'audiovisuel en France. Dans le domaine de la télévision, il s'agit de TF1 (TF1 Vidéo et MyTF1VOD; e-TF1 et direction générale), France Télévisions (direction générale), M6 (M6 Web) et Canal+ (service gérant les recommandations). Ont été aussi rencontrés des responsables du service VOD du principal fournisseur d'accès à Internet (Orange VOD) ainsi que de la filiale Plurimédia (gestion et métadonnées) du groupe Lagardère ${ }^{13}$. Les entretiens portaient sur le fonctionnement et le positionnement des services dans les groupes, les modèles d'affaires mis en œuvre ou recherchés, la prise en charge et la construction de différentes modalités de recommandation, ainsi que sur la connaissance et la gestion des parcours des consommateurs.

En prolongement des entretiens, nous avons procédé à une analyse des dispositifs sociotechniques mettant à disposition des catalogues de contenus audiovisuels, dans leurs versions web,

\footnotetext{
12 Un service de partage de vidéos comme YouTube préfère, à une intervention préalable sur les contenus, une recommandation contributive fondée sur l'agrégation des jugements des utilisateurs (likes, commentaires, etc.).

${ }^{13}$ La plupart des personnes rencontrées ayant souhaité l'anonymat et la confidentialité, nous ne mentionnerons pas leurs fonctions et rendrons compte de manière indirecte de leurs propos.
}

tic\&société - 10(2-3), 2016-2017 
Une question de comportement. Recommandation des contenus audiovisuels et transformations numériques

destinées aux téléviseurs ou aux supports mobiles (à l'exception de Plurimédia), comme MyTF1 et MyTF1VOD, FranceTV Pluzz et FranceTV Pluzz VàD, 6Play et M6VOD, CanalPlay, CanalPlay VOD et Canal+ à la demande, Orange VOD, Netflix et le service indépendant FilmoTV. Cette analyse visait à étudier l'architecture et le fonctionnement du dispositif, les lignes éditoriales, les appels à contribution, les différentes formes de personnalisation, les manières dont ces dispositifs cadrent et encadrent les pratiques.

\section{$2.1 \mathrm{La}$ recommandation éditoriale: juger n'est pas (toujours) prescrire}

Sous différentes formes et à des degrés divers, les dispositifs étudiés sont l'occasion pour les entreprises de renouveler la présentation de leur savoir-faire en matière de programmation, en affirmant certaines propositions propres ou en rappelant leur identité. Au-delà des environnements visuels et de contenus communs, la recommandation éditoriale permet de maintenir le consommateur dans un rapport déjà connu avec un opérateur. Ainsi, ces dispositifs bénéficient-ils de la visibilité et de la reconnaissance liées aux marques, aux lignes éditoriales, aux types de programmations et aux contenus directement associés à un groupe (notamment dans le cas des programmes de flux). L'observation des dispositifs déployés et les entretiens ont été l'occasion de constater le lien très fort entre l'expertise de programmation, le positionnement éditorial et la présentation des catalogues. L'exemple du dispositif de télévision de rattrapage de France Télévisions (FranceTV Pluzz) illustre le lien direct, paroxystique, entre les contenus proposés et les chaînes (dont la linéarité est reproduite pour présenter les contenus) : au moment du lancement du service, la question de la "personnalisation " ne se posait pas, et le choix a été fait de ne pas privilégier des contenus ou des chaînes plus que d'autres ${ }^{14}$. Dans le même temps, et de manière peut-être contradictoire, certains contenus particuliers étaient mis en avant ("Nos recommandations ») sans que les critères de choix ne soient expliqués au consommateur.

${ }^{14}$ Entretien avec un/une responsable de France Télévisions. 
La recommandation peut également se construire sur la base de catégories dont la chaîne estime qu'elle correspond à son image ou à sa programmation. Cette recommandation se fait en lien au contenu (comme dans l'exemple de MyTF1 VOD), où sont proposés des regroupements non pas par genres mais par sortes de thématique, ou même d'atmosphères, avec des titres comme " ils sont fous ces Anglais " ou "sexe sans complexe ". Elle peut contenir des séries, des films, des émissions de plateau... La recommandation peut aussi faire appel à d'autres types de regroupements, par exemple une sélection de films autour d'une actrice.

La recommandation éditoriale sous la forme de regroupements thématiques apparaît de manière particulière dans le cas du groupe Canal+. Une distinction apparente y est faite entre le service de VOD à l'unité, avec des catégorisations grossières par genre ou la mention de "coups de cœur », et le service de VOD par abonnement, marqué par la présence d'une éditorialisation plus poussée, avec des sélections assumées ${ }^{15}$. Celles-ci sont justifiées par la volonté de défendre et de promouvoir le rapport particulier du groupe au cinéma: d'après notre enquête, elles ne semblent cependant pas être jugées déterminantes ${ }^{16}$. La recommandation éditoriale vient simplement contribuer à la représentation qualitative d'une offre de contenus, à la différence d'une proposition qui ne serait qu'algorithmique.

Au-delà de recommandations portant la marque de l'entreprise, on observe certaines interventions éditoriales plus précises, qui présentent le catalogue sous un jour particulier (informations sur l'univers de l'auteur, approfondissements thématiques, etc.). Ainsi, le groupe M6 a développé au sein de son service de télévision de rattrapage 6Play plusieurs ensembles de contenus réunis autour de thèmes identifiés (mode, cuisine, immobilier, etc.), avec des personnalités reconnues, présentes sur les antennes du groupe:

\footnotetext{
${ }^{15}$ Par exemple, une mise en lien avec le Festival de Cannes, auquel le groupe est traditionnellement associé.

${ }^{16}$ Entretien avec un/une responsable de Canal+.
}

tic\&société - 10(2-3), 2016-2017 
Une question de comportement. Recommandation des contenus audiovisuels et transformations numériques

s'affirme ainsi la volonté de développer davantage de contenus, ce qui nécessite la création d'un véhicule plus large, une «métamarque », ce qu'est 6 Play ${ }^{17}$.

L'intervention des opérateurs auprès du consommateur est jugée par nos interlocuteurs comme essentielle, car elle est une des meilleures façons pour l'entreprise d'affirmer sa différence par rapport à ses concurrents. Si l'importance de l'éditorialisation a été mise en avant pour guider le consommateur, ce n'est pourtant pas dans une logique de conversion immédiate en achat ou location de contenus. Un responsable d'Orange, par exemple, nous a expliqué que les observations des parcours des consommateurs montraient que ces recommandations éditoriales n'ont un impact que sur un nombre limité $(5 \%)$ des consommations. Pour autant, l'importance de ces présentations est défendue parce qu'elle contribue de manière significative à l'environnement global du service, notamment pour les contenus cinématographiques. Ainsi, la recommandation éditoriale apparaît-elle aux professionnels comme un moyen d'améliorer la qualité globale du dispositif tel qu'il est perçu par les consommateurs, même s'ils n'y ont que faiblement recours.

La volonté d'affirmer son identité explique sans doute pourquoi le choix de l'éditorialisation est largement celui d'un certain nombre d'acteurs indépendants, dont les catalogues sont accessibles sur le web ou sur certaines set-top-boxes et offres de fournisseurs d'accès à Internet. L'exemple du site de VOD FilmoTV illustre les possibilités d'une recommandation éditoriale poussée, avec à la fois des connaissances factuelles sur le film, un résumé et des éléments critiques émanant d'une chroniqueuse identifiée. La volonté est ici clairement d'affirmer une démarche artisanale et de se différencier par rapport aux offres de recommandation jugées plus industrielles.

${ }^{17}$ Entretien avec un/une responsable de M6. 
Joëlle FARCHY, Cécile MÉADEL, Arnaud ANCIAUX

\subsection{Le silence paradoxal des recommandations des internautes}

La plupart des entreprises audiovisuelles ont ouvert des possibilités techniques d'accueil des contributions des internautes. Ces dispositifs ne recueillent cependant qu'un nombre peu élevé de contributions élaborées. Ainsi, le site de vidéos Canalplay VOD (page "bouche-à-oreille ») vise à encourager et à organiser le recueil et la publication de recommandation contributive; il va jusqu'à leur promettre une éventuelle rétribution financière, signe que ces contributions sont difficiles à obtenir. Le site intègre aussi des fonctionnalités permettant des liens avec les réseaux sociaux, mais, outre que celles-ci sont faiblement assumées par crainte de perdre le contrôle sur les contributions, elles ne sont pas jugées déterminantes par les responsables du service, tout comme elles sont faiblement utilisées par les consommateurs ${ }^{18}$.

La composante arithmétique de la recommandation apparaît plus importante dans les dispositifs étudiés. II ne s'agit plus de recueillir l'expression des internautes, mais des informations sommaires (notes et étoiles, likes, partages, etc.) permettant, à partir de l'opinion individuelle des consommateurs sur les contenus, de formuler des propositions collectives. Des classements («top des spectateurs", «meilleures notes spectateurs », etc.) sont présentés aux côtés des classements similaires issus de la presse (reprenant directement la dichotomie proposée par AlloCiné).

Dans les dispositifs étudiés, la dimension contributive, lorsqu'elle existe, est en effet fréquemment le résultat d'une importation dans le dispositif d'avis provenant d'acteurs extérieurs beaucoup plus efficaces dans l'économie de la contribution, tels AlloCiné ou SensCritique ${ }^{19}$. Comme pour les contributions expressives, les entretiens ont montré que les notes et les étoiles

\footnotetext{
${ }^{18}$ Entretien avec un/une responsable de Canal+.

${ }^{19}$ Celui-ci est ainsi visible dans l'exemple de Filmo TV, où un onglet intègre, aux côtes de l'avis de journalistes, ceux d'internautes ayant publié des critiques sur la plateforme SensCritique.
}

tic\&société - 10(2-3), 2016-2017 
Une question de comportement. Recommandation des contenus audiovisuels et transformations numériques

sont peu utilisées par les consommateurs, quand elles ne sont pas simplement mises de côté dans la conception des dispositifs ${ }^{20}$. Un grand nombre des systèmes de notations doivent donc, là encore, importer et intégrer des systèmes de notes et d'étoiles développés et synthétisés par d'autres acteurs. Les contributions des internautes sont ainsi en grande partie sous-traitées à des acteurs externes spécialisés.

De la même façon que les avis et les contributions des internautes sollicités par Allociné ne sont au final pas véritablement utilisés par la plate-forme (Pasquier, Beaudouin et Legon, 2014), celles mises en ligne par les entreprises audiovisuelles participent peu au renouvellement de leur modèle économique. La recommandation contributive n'apparaît réellement ni comme un élément de différenciation concurrentielle ni, selon les acteurs interviewés, comme un moyen de susciter des actes de consommation. La valorisation de l'usager et de sa participation active, souvent évoquée dans la littérature (voir supra, partie 1), relève donc plus d'une promesse inscrite dans un contexte sociodiscursif et économique que d'une dynamique impulsée par les acteurs historiques et concrétisée dans des dispositifs efficaces. Ainsi, les stratégies déployées par différents acteurs de l'écosystème de l'audiovisuel demeurent en grande partie construites autour des logiques propres aux offres spécifiques des acteurs et aux savoir-faire qu'ils maîtrisent.

À l'opposé de la recommandation contributive peu mobilisée, l'intervention des internautes semble plus susceptible d'être directement valorisée par les opérateurs lorsqu'il s'agit de recommandations autoentretenues fondées sur les comportements plutôt que sur les jugements. La présentation classique des meilleures ventes au sein des dispositifs apparaît comme un moyen central pour susciter l'achat ou la location de contenus : l'un des cas observés a d'ailleurs supprimé les classements reposant sur des jugements et des critiques (« top presse », « top

${ }^{20}$ Entretien avec un/une responsable de TF1. 
spectateurs »), pour ne conserver qu'un «top des ventes ${ }^{21}$. Si ce dispositif peut paraître particulièrement fruste, nos interlocuteurs signalent que c'est grâce à cette présentation que se fait la plus grande partie des actes de consommation (jusqu'à près de $80 \%$ des locations dans le cas de l'un des dispositifs, ou $90 \%$ des ventes sur le top 50 des nouveautés pour certains services de VOD à l'acte). Sous cette forme simplifiée, la recommandation aiguille le choix d'un grand nombre de consommateurs qui, plutôt que de se laisser enfermer dans des recommandations qu'on lui aurait faites, préfère savoir ce qu'aiment les autres pour se positionner ${ }^{22}$.

Au final, la recommandation issue des internautes se fait dans un silence paradoxal: les internautes "écoutent " ce que font leurs pairs en matière d'achats, et non pas ce qu'ils "disent" (contributions expressives ou étoiles); pour le dire autrement, les comportements priment sur les opinions. Ce constat est exacerbé dans les dispositifs de recommandation personnalisée.

\subsection{Les atouts de la recommandation personnalisée}

La recommandation personnalisée se retrouve dans la quasitotalité des dispositifs étudiés, selon des degrés de précision et de lien avec les contenus initialement consultés qui varient. Les métadonnées, qu'elles soient ou non visibles pour les utilisateurs du dispositif, servent à réaliser des propositions en lien avec l'intérêt manifesté au travers de la consultation d'un contenu. Elles peuvent s'appuyer sur le genre, les acteurs, les réalisateurs, les époques, etc., et se présentent sous la forme d'espaces introduits par des éléments de catégories et de genres, ou des interpellations comme "vous aimerez aussi » ou "à voir également ». Les comportements sont révélés sur la base des données à caractère personnel récoltées au sein ou autour du dispositif. Dans un premier temps, il s'agit d'inciter le consommateur à déclarer un certain nombre d'éléments (données

\footnotetext{
${ }^{21}$ Entretien avec un/une responsable d'Orange.

${ }^{22}$ Idem.
}

tic\&société - 10(2-3), 2016-2017 
Une question de comportement. Recommandation des contenus audiovisuels et transformations numériques

à caractère objectif, comme l'âge, le sexe, l'identité civile, la composition du foyer, ou à caractère subjectif, comme des préférences de contenus, de genres, etc.) afin qu'il organise par lui-même une partie de sa consommation, et ce, dès l'entrée dans le dispositif, nécessitant généralement une inscription. Des réponses à quelques questions permettant d'établir des segmentations de la consommation peuvent être obtenues de manière ludique et rapide (par exemple, Netflix propose un simple choix d'images, 6Play offre de choisir des préférences plus larges, aboutissant rapidement aux contenus existants). Dans un second temps, des données sont récoltées grâce à l'observation des parcours de consommation afin de déterminer des comportements attribuables au consommateur, à différents membres du foyer, etc. Les dispositifs étudiés varient de manière importante, certains, comme Canal+, ne demandant aucune information déclarative directement au consommateur. D'autres, comme Orange, recueillent beaucoup de données sur les usages, mais ne construisent que peu de données de profil (entendues au sens où aucune préférence n'est demandée aux utilisateurs sur leurs genres favoris) : la recommandation est alors « diffuse $»^{23}$.

Plusieurs de nos interlocuteurs estiment que la logique d'un suivi des consommateurs pourrait s'avérer contre-productive si elle aboutissait à briser la confiance et l'image du groupe, sur un sujet devenu grand public. L'observation des dispositifs concernés souligne d'ailleurs un relatif effacement d'une personnalisation qui, si elle se donne comme objectifs de satisfaire le consommateur intéressé et de répondre à des impératifs du dispositif, ne doit pas être perçue comme une modalité de surveillance.

Pour fournir des recommandations, même sous une forme algorithmique, les dispositifs doivent opérer une qualification préliminaire des contenus et des utilisateurs qui ne peut être intégralement automatisée. Figure d'abord la catégorisation, généralement effectuée par genres avec des degrés de granularité fort divers, regroupant nouveautés et derniers ajouts et reposant

${ }^{23}$ Entretien avec un/une responsable d'Orange. 
sur un certain nombre de mots-clés et métadonnées. La qualification des contenus fait l'objet pour Netflix d'une intense activité en interne difficile à appréhender pour le chercheur. Pour d'autres acteurs, la production des métadonnées pour catégoriser les contenus est l'objet d'une spécialisation: l'entreprise Plurimédia propose ainsi l'élaboration de guides numériques, fournissant images et vidéos, bandes-annonces, résumés dans des formats compatibles avec les dispositifs des entreprises audiovisuelles. Les informations en provenance d'AlloCiné proposent des métadonnées formatées et «transportées techniquement " au sein des dispositifs parmi d'autres métadonnées par un service comme celui proposé par Plurimédia ${ }^{24}$. La production et la gestion de ces données font très largement l'objet d'une sous-traitance ou de relations complexes. Ainsi, Plurimédia entretient des liens avec le moteur développé par l'entreprise Spideo, lui-même intégré dans le dispositif CanalPlay.

Ensuite, la recommandation personnalisée fondée sur l'analyse détaillée des comportements des internautes se révèle particulièrement efficace. Comme le résume Dominique Cardon (2015) : " La logique algorithmique colle à ce que font les individus en considérant, de façon très conservatrice, qu'ils sont rarement à la hauteur de leurs désirs. En préférant les conduites aux aspirations, les algorithmes nous imposent ce réalisme efficace " (p. 70). La recommandation personnalisée fait sienne un constat traditionnel des études d'audience (Méadel, 2010) selon lequel existe un écart entre ce que les consommateurs déclarent aimer et ce qu'ils regardent en fait. Aimer un contenu, vouloir qu'il existe et soit diffusé ne conduit pas nécessairement à le regarder.

Les entretiens réalisés nous ont permis de mettre en lumière l'importance d'une autre source d'efficacité de cette forme de recommandation. Comme nous l'ont expliqué des responsables de plates formes, l'objectif est de pousser des contenus vers le

${ }^{24}$ Entretien avec un/une responsable de Plurimédia.

tic\&société - 10(2-3), 2016-2017 
Une question de comportement. Recommandation des contenus audiovisuels et transformations numériques

consommateur, ce dernier ne pouvant obtenir du catalogue suffisamment de contenus l'intéressant a priori ${ }^{25}$.

L'efficacité de la recommandation personnalisée repose sur le fait qu'elle permet de résoudre le paradoxe de l'incomplétude de l'offre, c'est-à-dire de la finitude des catalogues face à la promesse d'une offre numérique illimitée. En effet, bien que les discours marketing développés autour du lancement des services de vidéos numériques à partir des années 2000 aient promu l'idée que les consommateurs allaient avoir accès à tous les contenus les intéressant, au moment et à l'endroit qu'ils souhaitaient, paradoxalement, les offres de vidéos à la demande, et notamment celles sur abonnement, sont construites sur des catalogues de droits limités (en raison de la concurrence, d'accords partiels avec les détenteurs des droits, des logiques d'exclusivité, de certaines réglementations, etc.). Dès lors, la recommandation personnalisée limite l'attention et la recherche du consommateur aux seules préférences correspondant aux droits détenus par l'opérateur du dispositif.

L'enjeu peut être résumé par un exemple simple : imaginons un consommateur ayant un intérêt marqué pour mille films dans l'absolu et une plateforme sur abonnement qui possède des droits pour cent de ces films seulement. Si le consommateur cherche par lui-même les films qui l'intéressent, il risque d'être déçu neuf fois sur dix (il ne trouvera pas les neuf cents films qui l'intéressent et dont la plateforme ne détient pas les droits), ce qui suscitera un sentiment d'insatisfaction. L'enjeu pour la plateforme est donc, à catalogue constant, de lui proposer l'un après l'autre chacun des cent films l'intéressant pour lesquels elle possède les droits. Dans les deux cas, avec ou sans recommandation personnalisée, le nombre de films à la fois intéressants et accessibles, l'utilité absolue pour le consommateur, reste identique, mais sa perception peut en être changée dans le second cas. Autrement dit, la recommandation doit permettre d'organiser le décalage, suffisamment important pour provoquer une sortie du dispositif,

${ }^{25}$ Entretien avec un/une responsable d'Orange. 
entre la promesse que le consommateur pourra trouver tout ce qu'il souhaite et le constat que le catalogue ne peut répondre qu'à une partie déterminée et limitée de ses souhaits.

\section{Conclusion : restructurations des marcEés et renouvellement des métiers}

Alors que jamais n'a été disponible une telle profusion de contenus audiovisuels, les acteurs historiques du secteur se retrouvent aux marges d'une vaste restructuration des marchés dans laquelle les dispositifs de recommandation apparaissent centraux. Ce n'est pas le moindre des paradoxes des évolutions en cours. Chacune à leur manière, les diverses formes de recommandation posent en effet aux opérateurs de mise à disposition de contenus audiovisuels en ligne de nouveaux défis qui nécessiteront de tester des formes de différenciation concurrentielle.

\section{Des recommandations éditoriales marginalisées dans un monde délinéarisé et individualisé}

La construction de la recommandation éditoriale est le prolongement direct, presque naturel, de l'une des fonctions traditionnellement attachées aux chaînes de télévision linéaire, à savoir la maîtrise des mécanismes de programmation, visant à rassembler le plus grand nombre de téléspectateurs autour d'un contenu pendant une période donnée (dans le cas de la vidéo commerciale gratuite financée par la publicité) ou à augmenter la satisfaction des téléspectateurs à l'égard des contenus proposés (dans le cas de la vidéo payante). Dans le marché de l'audiovisuel, c'était traditionnellement le travail des chaînes que de guider le choix du consommateur mis en ordre, le nom était bien choisi, par des "grilles de programme ", avec une hiérarchisation temporelle entre les périodes (prime time, week-end, matinée, jours fériés, etc.), des spécificités horaires ou des distinctions entre les programmes.

La délinéarisation, qui englobe le libre choix de l'horaire, du programme, de la manière de consommer (intégrale/partielle,

tic\&société - 10(2-3), 2016-2017 
Une question de comportement. Recommandation des contenus audiovisuels et transformations numériques

accélérée/ralentie, interrompue/continue) et du support (téléviseur, ordinateur, téléphone, tablette) bouscule cet ordre et rouvre les manières dont s'opère la rencontre entre offre et demande. De la maîtrise de la programmation de plages horaires avec des contenus particuliers, la création de rendez-vous reconnus par les consommateurs, et l'éventuelle recherche d'un cumul d'audience valorisé sur le marché publicitaire, la fonction des acteurs se déplace - au moins en partie - vers la constitution, la gestion et la promotion d'un catalogue de vidéos, disponibles pendant un temps, d'une longueur définie, et susceptibles ou non d'exclusivités.

La recommandation éditoriale reste le cœur de métier des opérateurs historiques ainsi qu'une forme de recommandation particulièrement valorisée par des pure players indépendants de I'Internet. Cependant, les tentatives de renouvellement des entreprises, qui les conduisent à chercher à tâtons les meilleures manières de susciter de l'intérêt et de faire la différence, reflètent l'apparente contradiction entre le principe même de la recommandation éditoriale et le monde délinéarisé et individualisé dans lequel ces dispositifs s'inscrivent. Sans être amenée à disparaître, la recommandation éditoriale est appelée à se renouveler profondément; en effet, de nouvelles formes de recommandation émergent, contributives ou personnalisées, a priori plus adaptées au nouvel environnement numérique.

\section{Des recommandations contributives qui échappent aux entreprises audiovisuelles}

Les dispositifs étudiés dans le cadre de cet article montrent que, contrairement aux attentes suscitées, les dispositifs de recommandation contributive, lorsqu'ils sont proposés par les opérateurs, sont en réalité peu mobilisés par les internautes (voir supra). Une grande partie de l'activité de contribution de ces derniers échappe en effet aux entreprises du secteur, en se déployant dans des espaces personnels ou collaboratifs propres: blogues d'amateurs, pages Facebook autour d'un contenu, sites de partage, collectifs d'échange, de sous-titrages, forums de fans, etc. (Béliard, 2016.) Les opérateurs de mise à disposition de 
programmes sont donc condamnés à importer des contributions effectuées auprès d'acteurs extérieurs spécialisés.

Cette forme d'externalisation, assumée par certains de nos interlocuteurs, leur pose cependant des problèmes de dépendance par rapport aux prestataires techniques ou aux réseaux sociaux comme Facebook. Un relatif abandon d'une partie de la connaissance et des rapports aux consommateurs au profit d'autres acteurs favorise de nouvelles formes de compétition. Ainsi, différents espaces d'échange sur les contenus audiovisuels et cinématographiques développent une concurrence pour l'attention directe. Autrement dit, les entreprises audiovisuelles voient leurs fonctions se limiter de plus en plus à la gestion d'un catalogue de contenus, tandis que les choix, la détermination des préférences et des qualités se font ailleurs.

\section{Des recommandations comportementales en débat}

Les recommandations autoentretenues, bien que relativement efficaces pour aiguiller les choix des internautes (voir supra), se révèlent particulièrement frustes et peu susceptibles de renouveler les modèles économiques du numérique.

La recommandation personnalisée algorithmique, au cœur de propositions ciblant les habitudes des consommateurs et historiques de consultation, malgré son efficacité, entraîne au contraire de grandes transformations et suscite un certain nombre d'interrogations. Parmi celles les plus usuellement formulées, on trouve l'idée de l'enfermement du consommateur dans ses habitudes. Ce risque de "bulle " (filter bubble) (Pariser, 2011) fait débat depuis les premiers travaux sur le sujet (Fleder et Hosanagar, 2009). Depuis bien longtemps, les capacités du web à confronter les internautes à des opinions divergentes, ou, pour le dire en d'autres termes, à contribuer à nourrir un espace public habermassien, fait question (Turkle, 1997 ; Sustein, 2001). Le même reproche est régulièrement fait à Facebook, accusé de fermer la fenêtre des internautes sur le monde en réduisant leur univers de choix et de relations. Bakshy, Messing et Adamic (2015) ont confirmé cette tendance à l'homophilie pour le réseau social à propos des opinions politiques. Ainsi, si l'on suit cette 


\section{Une question de comportement. Recommandation des contenus audiovisuels et transformations numériques}

approche controversée sur la bulle, l'algorithme ne ferait au mieux que renforcer le comportement des internautes : leur propension à ne fréquenter que des objets, des personnes, des idées conformes à leurs propres goûts et opinions; et leur rejet du nouveau, du différent, de l'inconnu.

Dans le domaine de l'audiovisuel, le risque d'enfermement pourrait aller jusqu'à la caricature consistant à proposer à chacun sa propre chaîne adaptée à ses goûts selon ses choix antérieurs. Dans ce cas, l'enjeu est celui de la diversité culturelle et d'une économie façonnée par une logique de la demande plutôt que par une logique de l'offre. Les propos du président du CSA illustrent cette crainte :

"L'algorithme présente certains risques: outre bien sûr le profilage, l'enfermement dans ses propres goûts au détriment de la découverte, la tentation de manipulations destinées à favoriser les œuvres produites par l'éditeur, voire, perspective particulièrement inquiétante, aujourd'hui encore utopique, la menace d'une standardisation de la création qui serait dictée par les attentes du public. Ces risques pour la diversité culturelle peuvent toutefois être atténués si les algorithmes ne sont pas exclusivement centrés sur les données fournies par les consommateurs, mais inspirés par les caractéristiques des œuvres proposées, par un travail sur les métadonnées associées à ces œuvres ${ }^{26}$.

Cependant, le rôle de l'algorithme dans l'enfermement et la constitution d'un « entre soi » mérite d'être nuancé. Le marché n'a pas attendu les algorithmes pour être à l'écoute des attentes du public, et l'impact du réseau ou de la reproduction sociale sur les pratiques de chaque individu est un débat que les travaux pionniers de Pierre Bourdieu n'ont toujours pas épuisé. De plus, l'impérieuse obligation pour l'offre de se renouveler sous peine de lasser conduit à des recommandations en devenir centrées sur des propositions de "découvertes " afin de pousser le spectateur hors de ses sentiers trop connus.

Au vu des dispositifs étudiés dans le cadre de cet article, plus que le risque d'enfermement, le problème le plus important posé

\footnotetext{
${ }^{26}$ Allocution d'Olivier Schrameck, Président du Conseil Supérieur de l'Audiovisuel, au Forum de Tokyo. 2 décembre 2014.
} 
par la recommandation algorithmique semble plutôt lié à l'absence de savoir-faire et à la perte de la relation client que ce nouvel outil induit pour les acteurs impliqués dans la mise à disposition de contenus. Les recommandations comportementales étudiées sont au cœur des stratégies des acteurs du numérique pour acquérir, gérer et valoriser les données personnelles (Anciaux et Farchy, 2015). La plus importante chaîne privée française, TF1, a signé en 2013 un accord de partenariat avec le réseau social Facebook afin de bénéficier des données et des outils dédiés au suivi et à l'analyse des conversations des internautes sur les programmes de la chaîne. De la même façon, le New York Times ou la BBC n'ont pas accès aux données sur leurs propres contenus circulant sur le réseau social. Tandis que ces opérateurs de contenus travaillent "à l'aveugle ", de nouveaux métiers apparaissent précisément centrés sur l'analyse de plus en plus sophistiquée des comportements d'usagers. La menace sur la diversité culturelle proviendra peut-être moins de la toute-puissance des algorithmes que de l'éventuel pouvoir oligopolistique qu'un petit nombre d'acteurs maîtrisant les données de comportements des consommateurs pourraient construire.

\section{RÉFÉRENCES BIBLIOGRAPHIQUES}

AKRICH M. et C. MÉADEL, 2006, «Télévision à la carte, un divorce annoncé ", Réseaux, vol. 5, n 139, pp. 75-103.

AMATRIAIN X. et J. BASILICO, 2015, «Recommender Systems in Industry: A Netflix Case Study ». Recommender Systems Handbook, New York, Springer, pp. 385-419.

ANCIAUX A., 2014, Réinventer l'économie du journalisme. OuestFrance et Québecor, Thèse de doctorat, Université de Rennes 1 - Université Laval.

ANCIAUX A. et J. FARCHY, 2015, « Données personnelles et droit de propriété : quatre chantiers et un enterrement », Revue internationale de droit économique, vol. XXIX, n 3 , pp. 307331.

tic\&société - 10(2-3), 2016-2017 
Une question de comportement. Recommandation des contenus audiovisuels et transformations numériques

ASSOGBA H., A. COUTANT, J.-C. DOMENGET et G. LATZOTOTH, 2016, "Les publics imaginés et réels des professionnels d'internet », Communication, vol. 33, $\mathrm{n}^{\circ} 2$.

BAKSHY E., S. MESSING et L. A. ADAMIC, 2015, "Exposure to Ideologically Diverse News and Opinion on Facebook ", Science, vol. 348, $\mathrm{n}^{\circ}$ 6239, pp. 1130-1132.

BEAUVISAGE T., J.-S. BEUSCART, V. CARDON, K. MELLET et M. TRESPEUCH, 2013, "Notes et avis des consommateurs sur le web », Réseaux, vol. 1, $\mathrm{n}^{\circ} 177$, pp. 131-161.

BÉLIARD A.-S., 2016, S'il te plaît, dessine-moi une série. Contributions des internautes et séries télévisées, Paris, Presses des Mines.

BENKLER Y., 2006, The Wealth of Networks: How Social Production Transforms Markets and Freedom, London, Yale University Press.

BEUSCART J.-S., T. BEAUVISAGE et S. MAILLARD, 2012, « La fin de la télévision ? Recomposition et synchronisation des audiences de la télévision de rattrapage », Réseaux, vol. 5, $\mathrm{n}^{\circ} 175$, pp. 43-82.

CALLON M., C. MÉADEL et V. RABEHARISOA, 2000, "L'économie des qualités », Politix, vol. 13, n 59, pp. 211239.

CARDON D., 2015, À quoi rêvent les algorithmes: nos vies à l'heure des big data, Paris, Seuil.

CARDON D. et A. CASILLI, 2015, Qu'est-ce que le digital labor?, Paris, INA.

CHA J. et S. M. CHAN-OLMSTED, 2012, "Substitutability Between Online Video Platforms and Television », Journalism \& Mass Communication Quarterly, vol. 89, pp. 261-278.

COLLIN P. et N. COLIN, 2013, Mission d'expertise sur la fiscalité de l'économie numérique, Rapport au ministre de l'Économie et des Finances, France.

DUJARIER M.-A., 2008, Le travail du consommateur : de McDo à Ebay, comment nous coproduisons ce que nous achetons, Paris, La Découverte. 
Joëlle FARCHY, Cécile MÉADEL, Arnaud ANCIAUX

ELKHATIB Y., R. KILLICK, M. MU et N. RACE, 2014, "Just Browsing?: Understanding User Journeys in Online TV », Proceedings of the ACM International Conference on Multimedia, ACM.

FARCHY J., C. MÉADEL et G. SIRE, 2015, La gratuité, à quel prix ?, Paris, Presses des Mines.

FARCHY J. et F. MOREAU, 2016, L'économie numérique de la distribution des œuvres et le financement de la création, Conseil supérieur de la propriété littéraire et artistique.

FLEDER D. et K. HOSANAGAR, 2009, «Blockbuster Culture's Next Rise or Fall: The Impact of Recommender Systems on Sales Diversity ", Management Science, vol. 55, $\mathrm{n}^{\circ}$ 5, pp. 697-712.

GOMEZ-URIBE C. A. et N. HUNT, 2015, « The Netflix Recommender System: Algorithms, Business Value, and Innovation ", Transactions on Management Information Systems, ACM, vol. 6, $\mathrm{n}^{\circ}$ 4, pp. 1-19.

LEE C. Y., C. K. HONG et K. Y. LEE, 2010, "Reducing Channel Zapping Time in IPTV Based on User's Channel Selection Behaviors ", IEEE Transactions on Broadcasting, vol. 56, pp. 321-330.

MACHADO VIEIRA P. K. et N. CORREIA QUEIROZ LINO, 2015, "Recommendation of Multimedia Content in Digital Convergence Environments: TV and Web », Latin America Transactions, IEEE, vol. 13, pp. 3467-3475.

MADRIGAL A., 2014, "How Netflix Reverse Engineered Hollywood », The Atlantic, vol. 2.

MÉADEL C., F. MUSIANI et J. FARCHY, 2016, « Les alchimistes de la parole en ligne ", Communication, vol. 34, $n^{\circ} 1$.

MÉADEL C., 2010, Quantifier le public. Paris, Economica.

MOULIER-BOUTANG Y., 2010, L'abeille et l'économiste, Paris, Carnets Nord.

PARISER E., 2011, The Filter Bubble: What the Internet is Hiding From You, Londres, Penguin UK.

PASQUIER D., V. BEAUDOUIN et T. LEGON, 2014, " Moi je lui donne 5/5». Paradoxes de la critique amateur en ligne, Paris, Presses des Mines.

PATINO B., 2016. Télévisions, Paris, Grasset.

tic\&société - 10(2-3), 2016-2017 
Une question de comportement. Recommandation des contenus audiovisuels et transformations numériques

REAGLE J. M., 2015, Reading the Comments : Likers, Haters, and Manipulators at the Bottom of the Web, Cambridge, MIT Press.

REBILLARD F., 2011, "Création, contribution, recommandation : les strates du journalisme participatif », Les Cahiers du journalisme, $\mathrm{n}^{\circ} 22-23$, pp. 28-40.

RESNICK P. et H. R. VARIAN, 1997, "Recommender Systems ", Communications of the ACM, vol. 40, $\mathrm{n}^{\circ} 3$, pp. 56-58.

RICCI F., L. ROKACH et B. SHAPIRA, 2015, "Recommender Systems: Introduction and Challenges ", dans F. RICCl, L. ROKACH et B. SHAPIRA (dir.), Recommender Systems Handbook, Boston, Springer US, pp. 1-34.

ROCHET J.-C. et J. TIROLE, 2003, « Platform Competition in Twosided Markets ", Journal of the European Economic Association, vol. 1, $\mathrm{n}^{\circ} 4$, pp. 990-1029.

SCHERER É., 2015, «La TV demain: 10 enjeux de transformation », Méta-média. Cahier de tendances médias de France Télévisions, Printemps/Été.

SCHOLZ T. (dir.), 2013, Digital Labor: The Internet as Playground and Factory, Londres, Routledge.

SIMON H. A., 1971, " Designing Organizations for an Informationrich World », dans M. GREENBERGER (dir.), Computers, Communication, and the Public Interest, Baltimore, The Johns Hopkins Press, pp. 37-72.

SUSTEIN C., 2001, Republic.com, Princeton, Princeton University Press.

TERRANOVA T., 2000, "Free Labor: Producing Culture for the Digital Economy », Social Text, vol. 18, n², pp. 33-58.

TRYON C., 2015, « TV Got Better: Netflix's Original Programming Strategies and the On-Demand Television Transition », Media Industries, vol. 2, $\mathrm{n}^{\circ} 2$.

TURKLE S., 1997, Life on the Screen: Identity in the Age of the Internet, New York, Simon \& Schuster Trade.

VERDIER H. et N. COLIN, 2012, L'âge de la multitude. Entreprendre et gouverner après la révolution numérique, Paris, Armand Colin. 